\title{
Cellulose-Based Reflective Liquid Crystal Films as Optical Filters and Solar Gain Regulators
}

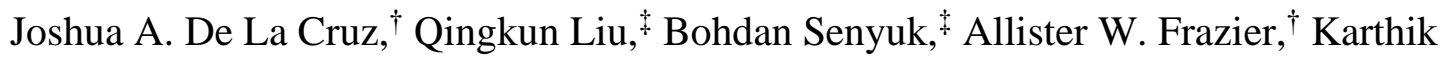 \\ Peddireddy, ${ }^{\ddagger}$ and Ivan I. Smalyukh ${ }^{*}, \dagger, \S, \S$
}

${ }^{\dagger}$ Materials Science and Engineering Program, University of Colorado, Boulder, Colorado 80309, United States

Department of Physics, University of Colorado, Boulder, Colorado 80309, United States

${ }^{\S}$ Soft Materials Research Center and Department of Electrical, Computer and Energy

Engineering, University of Colorado, Boulder, Colorado 80309, United States

${ }^{\perp}$ Renewable and Sustainable Energy Institute, National Renewable Energy Laboratory and University of Colorado, Boulder, Colorado 80303, United States

\begin{abstract}
Many promising approaches for designing interactions of synthetic materials with light involve solid optical monocrystals and nanofabricated photonic crystal structures with spatially periodic variations of refractive index. Although their high costs limit current technological applications, remarkably, such photonic and optically anisotropic materials have also evolved throughout nature and enable narrow or broad-band spectral reflection of light. Here we use self-assembly of biomaterial cellulose nanocrystals to obtain three-layer films with helicoidal and nematic-like organization of the cellulose nanoparticles, which mimics naturally occurring polarization-insensitive reflectors found in the wings of Plusiotis resplendens beetles. These films were characterized with polarized optical microscopy and circular dichroism spectrometry, as well as scanning and transmission electron microscopies. These films exhibit high reflectivity tunable within the visible and near-infrared regions of the optical spectrum and may find applications ranging from color filters to smart cloth designs and in solar-gainregulating building technologies.
\end{abstract}

KEYWORDS: photonic crystal, circular polarizer, tunable reflection, chiral nematic, biomaterials, liquid crystal 
Photonic crystals enable a host of practical applications such as nonlinear waveguides, ${ }^{1,2}$ advanced anticounterfeiting structures ${ }^{3,4}$ and low-energy-consuming displays $;, 5$ however, the bulk of these material systems are carefully prepared via top-down methods such as photo- and electron-lithography. Nanofabrication affords precise control over structure but is obtained at the cost of detrimentally impacting scalability and throughput, hampering economic feasibility, and ultimately limiting such materials' practical scope. Nonetheless, photonic crystals produced bottom up via self-assembly are abundant throughout biology, including those in aberrationcorrected spherical lenses found in the arm ossicles of the marine organism Ophiocoma wendtii and viewing-angle-independent selective reflecting exoskeletons of the beetle species Plusiotis resplendens. ${ }^{7-9}$ These self-assembled photonic structures have driven researchers to apply bottom-up manufacturing in developing other synthetic photonic crystal systems, resulting in the formation of novel synthetic photonic material systems such as photonic diodes, ${ }^{9}$ tunable organic lasers, ${ }^{10}$ and subpicosecond optical switches. ${ }^{11}$ The cost of these bottom-up crystal systems can further be decreased by utilizing common biomaterials such as cellulose. Cellulose is the most abundant biopolymer on the planet and is composed of polymerized D-glucose dimer chains arranged in a semicrystalline fashion. ${ }^{12}$ Cellulose nanocrystals (CNCs) are particularly interesting for photonic applications, as cellulose is an inexpensive renewable resource that can self-assemble into periodic optical planes serving as Bragg diffractors. ${ }^{13}$ However, the theoretical optical performance of traditional CNC-based systems is limited to $50 \%$ due to selective reflection of like-handed polarized radiation over opposite-handed polarized radiation, ${ }^{14}$ limiting their optical applications. This limitation thus far has only been overcome with the use of nonbiological-based retardation materials such as pentylcyanobiphenyl (5CB). ${ }^{9}$

In this work, we present the preparation, characterization, and optical modeling of a transparent, flexible, and economical CNC-based photonic composite structure serving as polarization-independent tunable Bragg reflectors operating within the visible and near-infrared (near-IR) regimes. CNCs derived from both bacteria cellulose and cotton cellulose are utilized in this study. The polarization-independent reflection of the laminar composite structure detailed herein is realized by insertion of a birefringent half-wave plate composed of nematic-like CNC between two reflective cholesteric-like $\mathrm{CNC}$-organosilica composite films, forming a laminar composite "sandwich structure" mimicking the exoskeletal of the aforementioned beetles. ${ }^{15}$ Electron microscopies reveal the spindle-like geometry of CNCs, and polarized optical 
microscopy shows their adoption of both nematic-like and cholesteric-like assemblies within the solid films, as controlled by our sample preparation conditions. The optical performance of the sandwich structure is contrasted with that of a single cholesteric-like film via reflective circular dichroism (CD) spectrometry and polarized optical microscopy (POM). The structure of the three layers is determined via scanning and transmission electron microscopies. Reflection spectra reveal a $\sim 95 \%$ peak reflectivity increase compared to a single film, with the total peak reflectivity of the structure reaching as high as $82 \%$. These CNC-based laminar composite photonic structures may enable applications such as low-emissivity and solar-gain-regulating films, color and transparent visible-infrared filters, smart fabrics, optically enabled document security features, optical isolators, and other applications where shielding radiative heat while preserving visible-range transparency is needed. Furthermore, the use of cheap, abundant biomaterials such as cellulose and the implementation of a bottom-up fabrication approach make these materials a feasible, cost-effective solution that is readily implemented.

\section{RESULTS}

Design and Physical Principles. A single left-handed cholesteric-like CNC film's theoretical $50 \%$ maximum reflection limit due to selective reflection is overcome by construction of a highly reflective and flexible composite $\mathrm{CNC}$-organosilica photonic structure, whose schematic is detailed in Figure 1a. The proposed photonic structure consists of two left-handed cholestericlike composite cotton-derived $\mathrm{CNC}$-organosilica films sandwiching an aligned nematic-like bacterial CNC optical retarder and possesses a polarization-independent reflection of incident radiation satisfying the Bragg diffraction condition. ${ }^{16}$ The three-layered approach to achieve total reflection from solely left-handed films is inspired by the beetle species Plusiotis resplendens, whose exoskeleton is composed of two left-handed helicoidal layers sandwiching a nematic-like assembly; all three of these layers are primarily composed of the same polysaccharide, chitin, the second most abundant biopolymer after cellulose ${ }^{17} \mathrm{CNCs}$ serve as a robust templating platform, imparting the cholesteric assembly onto soluble guest chemical species introduced to the colloidal dispersion,13 allowing the film's optical, mechanical, and chemical properties to be tailored; the cholesteric-like films' chemistry is optimized to maximize reflection, and the plasticizer polyethylene glycol ( $\mathrm{M}_{\mathrm{w}}: 400 \mathrm{~g} / \mathrm{mol}$, PEG-400) is added to improve flexibility and 
mechanical toughness. Unidirectional shearing can force CNCs' adoption of a nematic-like assembly, forming macroscopically aligned monodomain films, which serve as a birefringent phase retarder with designed retardation via a series of successive linear shear deposition cycles.

The polarization-independent reflection of the sandwich structure results from the combination of cholesteric- and nematic-like CNC optical elements, enabling reflection of both incident left-handed circularly polarized (LCP) light and righthanded circularly polarized (RCP) light solely by left-handed cholesteric-like CNC films. When natural light is incident upon the sandwich structure, the LCP component of light corresponding to the Bragg diffraction condition is selectively reflected. As incident RCP light is transmitted through the first cholesteric-like CNC film, it is converted into LCP light by the nematic-like CNC retarder acting as a half-wave plate. This LCP light is reflected by the second cholesteric-like layer and is converted back into RCP light as it transmits through the retarder again. Finally, this RCP radiation transmits through the first left-handed cholesteric-like film. Thus, we see all polarizations of light are reflected, as depicted in the scheme in Figure 1b. The reflective performance of the structure is polarizationindependent and shows 2-fold reflection enhancement as compared to a single cholesteric-like CNC film. Because the pitch of the cholesteric-like films and the retardation of the nematic-like film are tunable from the visible to near-IR regimes, it follows that this reflection enhancement is also tunable in those same regimes.

The flexible cholesteric-like CNC films' arbitrarily tunable reflection within the visible and near-IR regimes is achieved by controlling synthetic and processing conditions. Sulfuricacidcatalyzed hydrolysis of cellulose preferentially etches amorphous cellulose domains, yielding colloidal chiral cellulose nanocrystalline spindle-shaped rods. ${ }^{18}$ Different cellulose sources vary in their degree of crystallinity and size of crystallites, 16 and thus the size and yield of CNCs obtained strongly depend on their source. Colloidal stability is ensured by Coulombic repulsion between the negatively charged sulfate ester moieties of the CNCs. Concentrating above the CNC's critical Onsager concentration (about $3.5 \mathrm{wt} \%$ for bacterial CNCs and $5.0 \mathrm{wt}$ $\%$ for cotton $\mathrm{CNCs}$, depending on synthetic conditions and processing) results in a thermodynamically stable lyotropic liquid crystal (LC). ${ }^{19}$ The CNCs' inherit geometric chirality leads to adoption of the left-handed chiral nematic (cholesteric) LC phase with a helical axis denoted by $\chi$ (Figure 1 b). ${ }^{20,21}$ The cholesteric phase's twisting repeated optical planes of CNC spindle-shaped rods impart selective reflection of same-handed polarized radiation satisfying the 
diffraction condition ${ }^{16,22}$

$$
\lambda=\bar{n} p \cos \theta
$$

where $\lambda$ is the wavelength of reflected radiation, $\bar{n}$ is the average refractive index of the cholesteric medium (taken to be 1.5 for the $\mathrm{CNC}$-organosilica system), $p$ is the pitch of the medium, defined as the distance between two LC building blocks separated by a $2 \pi$ rotation, and $\theta$ is the angle of incidence with respect to the helical axis $\chi$ (normal to the plane of the film in our experiments). Cotton CNCs are used in the reflective layers of the sandwich structure due to their smaller size and thus smaller pitch compared to bacterial CNCs, blue-shifting their reflection into the visible and near-IR domains. The diffraction condition dictates reflection centered about a wavelength $\lambda_{0}=\bar{n} p$ with a full width at half-maximum $\Delta \lambda=\Delta n p,{ }^{23}$ with birefringence $\Delta n=\left(n_{\mathrm{e}}-\right.$ $n_{\mathrm{o}}$ ), where $n_{\mathrm{e}}, n_{\mathrm{o}}$ are the extraordinary and ordinary refractive indices of the birefringent medium, respectively. Colloidal CNCs preserve the cholesteric assembly upon evaporation in a process known as evaporation-induced self-assembly (EISA), ${ }^{24,25}$ forming freestanding lefthanded helicoidal dry films. Post-EISA the films undergo an according pitch shrinkage, blue-shifting the reflection peak of a dried film compared to its aqueous counterpart. ${ }^{13}$ Berreman $4 \times 4$ matrix simulation results, detailed below in the numerical modeling section, show reflectivity saturates at a film thickness of $\sim 10 p$, guiding us to prepare $10-15 \mu \mathrm{m}$ thick reflective films.

\section{Experimental Realization and Characterization of Tunable Single-Layer Reflective Films.}

Peak reflection of CNC-based films is readily tuned by increasing organosilica loading from 21.6 wt $\%$ to $32.6 \mathrm{wt} \%$ to increase its colloidal pitch, red-shifting its reflection wavelength from 400 $\mathrm{nm}$ to $915 \mathrm{~nm}$. Freestanding CNC-organosilica films prepared with $21.3 \mathrm{wt} \%$ (Table 1) organosilica loading exhibit $45 \%$ peak reflection of $400 \mathrm{~nm}$ light and appear blue in reflection (Figure 2a), while further increasing their loading to $24.0 \mathrm{wt} \%$ (Table 1) resulted in a $41.7 \%$ reflection of $557 \mathrm{~nm}$ light with a full width at half-maximum of $119 \mathrm{~nm}$, appearing green in reflection (Figure 2b). Further organosilica loading to $26.1 \mathrm{wt} \%$ (Table 1) formed a red reflective film (Figure 2c) reflecting 39.5\% of $688 \mathrm{~nm}$ light with a full width at half-maximum of $122 \mathrm{~nm}$, while loading near the maximum capable of sustaining the cholesteric phase results in a near-IR reflection with a peak reflection of $40.4 \%$ at $915 \mathrm{~nm}$ (Figure $2 \mathrm{~d}$ ). The films reflect $\sim 8 \%$ of background radiation due to the refractive index contrast of about 0.5 at both optical interfaces 
of the film. Macroscopic images of a single blue, green, and red reflective film are seen in the inset of Figure $2 \mathrm{~d}$, and enlarged images of the green and red reflective films are shown in Figure $2 \mathrm{e}$ and $\mathrm{f}$, respectively. The near-IR reflective film features $92 \%$ transparency averaged across the visible spectrum (Figure $2 \mathrm{~g}$ ) and excellent flexibility due to the addition of the plasticizer PEG400 (Figure 2h).

The uniform cholesteric-like CNC layers with magnetic field aligned single-domain organization are characterized by scanning electron microscopy (Figure 3). Maximum reflectivity of a single cholesteric-like $\mathrm{CNC}$ film per unit film thickness is attained by alignment of CNCs via subjection to an external static magnetic field during evaporation. CNCs are a negative magnetic susceptibility anisotropic material, and an external magnetic field of magnitude $\geq 2000 \mathrm{G}$ directed normal to the film's surface can overcome room-temperature thermal fluctuations, aligning the cholesteric LC with its helical axis $\chi$ parallel to the field, with the CNC director orthogonal to the field. ${ }^{24}$ Cross-sectional transmission electron micrographs confirm the static magnetic field successfully aligns the cholesteric-like domains of $\mathrm{CNC}$-organosilica composite structures, with domains with pitch as small as $286 \mathrm{~nm}$ (Figure 3a) being well aligned, corresponding to blue reflective films. This alignment technique is valid for films reflecting across the visible and near-IR regimes, as macroscopic-sized single-crystalline cholesteric-like domains corresponding to green-reflective films ( $p=357 \mathrm{~nm}$, Figure $3 \mathrm{~b}$ ), nearIR reflective films ( $p=660 \mathrm{~nm}$, Figure 3c), and films with even larger pitch $(p=1.3 \mu \mathrm{m}$, Figure 3d) are realized.

Cellulose-Based Retarders and Three-Layer Reflective Films. A tunable, unidirectionally aligned large-scale CNC nematic-like birefringent half-wavelength phase retarder plate is designed to convert incident RCP light into LCP light, nearly doubling reflectivity. It is manufactured by a series of successive linear shear deposition cycles and subsequent solvent evacuation. Shearing colloidal CNCs align the crystallites with the director along the shear direction; this arrangement is then locked into place by rapid evaporation of the colloid's solvent. These films introduce incident radiation to a retardation $L$ related to net birefringence $\Delta n$ and optical path length $d$ given by $L=\Delta n d$. The deposition of successive CNC layers discretely tunes the retardation of the birefringent plate by integer multiples of the retardation of a single layer.

This layer-by-layer manufacturing approach allows for preparation of optical retarders 
spanning the visible and near-IR ranges of wavelengths. Unidirectionally aligned, nematic-like organization of pure bacterial and cotton CNC films is confirmed by polarized optical and scanning electron microscopies, respectively. Minimal transmitted intensity between crossed polarizers is observed when the shearing direction is parallel to either polarizer (Figure 4a). Transmission between crossed polarizers is maximized when the $\mathrm{CNC}$ director is oriented $45^{\circ}$ with respect to either polarizer, confirming the presence of unidirectionally aligned CNCs, with their long axes oriented along the shear direction (Figure 4b).

The aligned CNC film features positive birefringence, as evidenced by insertion of a $530 \mathrm{~nm}$ retardation plate when the director is oriented $+45^{\circ}$ with respect to the polarizer, causing the overall system to appear bluish (Figure 4c). Scanning electron microscopy of the shear plane confirms both bacterial CNCs (Figure 4d) and cotton CNCs (Figure 4e) adopt the aligned nematic-like configuration with its director oriented along the shear direction. Nematic-like bacterial CNC-based wave plates with net birefringence $\Delta n=0.0226$ and retardation spanning 48 to $838 \mathrm{~nm}$ (Figure 4f) are formed, and the average retardation per bacterial CNC layer deposited is found to be $54 \mathrm{~nm}$. Bacterial CNCs are larger and retard radiation more than cotton CNCs, enabling course and fine adjustment of the wave plate's retardation. An average wavelength retardation $\Delta n d$ per unit film thickness $\mathrm{d}$ for bacterial CNCs is found to be $22.6 \mathrm{~nm}$ of retardation per micrometer of film thickness, compared to cotton CNCs at $6 \mathrm{~nm}$ of retardation per micrometer of film thickness.

Sandwiching the unidirectionally aligned nematic-like CNC wave plate between two reflective left-handed cholesteric-like CNC films increases reflectivity by as much as $95 \%$ compared to a single left-handed reflective film, due to its reflection of all polarizations of incident radiation satisfying the Bragg diffraction condition. Reflection and CD spectra of singlelayer films and their corresponding sandwich structure are recorded following the schematic detailed in Figure 5a. Spectrographical data revealing reflection performance of linearly polarized (LP) radiation show a single cholesteric-like $\mathrm{CNC}$-organosilica composite film reflected only $41.6 \%$ of peak radiation (occurring at a wavelength of $558.3 \mathrm{~nm}$ ), reaching $83.2 \%$ of its $50 \%$ reflection limit originating from selective reflection due to its left-handed chiral structure (Figure 5b). When LCP light is incident upon the visibly reflective lefthanded single film, a $63 \%$ increase of peak reflected radiation compared to incident LP light, as $67.8 \%$ reflection of incident $567.0 \mathrm{~nm}$ light, is observed (Figure 5b). This case contrasts the case when 
$\mathrm{RCP}$ radiation is incident upon the left-handed single film, in which case no reflection peak is detected (Figure 5b). A $\sim 10 \%$ background reflected intensity exists when RCP radiation is incident, due to the refractive index contrast of about 0.5 at both of the film's optical interfaces with air; when this $10 \%$ background reflection is subtracted from the LP and LCP curves, we calculate an $83 \%$ increase in reflected intensity for incident LCP radiation compared to incident LP radiation. This near doubling of the effective reflection is explained by the nature of LP radiation, which can be thought of as a superposition of in-phase LCP and RCP components. The polarization-dependent reflection of a single left-handed cholesteric-like film contrasts with the reflection of the sandwich structure, where polarization-independent reflection is achieved. A peak reflected intensity of $73.9 \%$ of incident $L P$ radiation at $537.4 \mathrm{~nm}$ by the sandwich structure is recorded (Figure 5c), corresponding to a $77.64 \%$ increase in LP reflection performance compared to a single reflective film. The LP reflective performance of the sandwich structure is similar to its performance when LCP radiation is incident, where a peak reflected intensity of $73.9 \%$ of $542.5 \mathrm{~nm}$ radiation is captured (Figure 5c). When combined with the RCP reflective performance of the sandwich structure, with a peak reflection of $72.2 \%$ at $534.0 \mathrm{~nm}$ (Figure $5 \mathrm{c}$ ), we see the sandwich structure reflects all polarizations of light equally, within experimental error. The $9 \mathrm{~nm}$ range of peak reflection of the single-layer film and sandwich structure arises from microstructural defects present within the sample. The reflective CD spectrum of each structure reinforces the contrast seen in the polarization-dependent nature of a single cholestericlike film compared to the sandwich structure (Figure 5e), as the polarization-dependent single film shows a nontrivial positive CD signal of $60.2 \%$ at $567 \mathrm{~nm}$, indicating LCP radiation interacts much more strongly with a single lefthanded film than RCP radiation. The CD signal of the sandwich structure confirms its polarization-independent interaction with light, as a very different $\mathrm{CD}$ response is obtained (Figure 5e).

The CD signal of the sandwich structure has a small peak of $11.0 \%$ at $567 \mathrm{~nm}$ (5.5 times decreased compared to a single film) and is caused by the $9 \mathrm{~nm}$ difference in LCP and RCP reflection peaks. Transmission spectra of single films and their corresponding sandwich structure are characterized by using an optical setup schematically shown in Figure 5d.

The corresponding transmission spectra of the single film and sandwich structure show similar transmission of natural light not satisfying the Bragg condition for both structures, except for the reflection trough at $567 \mathrm{~nm}$ (Figure 5f), which is found to be $66 \%$ lower for the sandwich 
structure compared to its single film counterpart.

The polarization-dependent interaction of light in a single visibly reflective film and the polarization-independent interaction of light of its corresponding visibly reflective sandwich structure are confirmed and visualized via reflective optical microscopy. A single reflective film (inset of Figure 6a) is seen to moderately reflect green LCP light (Figure 6a), while only marginally reflecting incident green LP light (Figure 6b). When RCP radiation is incident upon a single reflective film, about $10 \%$ of all light across the visible spectrum is reflected, and so the corresponding micrograph is dark gray (Figure 6c). The sandwich structure (inset of Figure 6d) reflects light with all polarizations of incident radiation equally, as reflective micrographs captured after LCP radiation is incident (Figure 6d) have similar color and intensity to micrographs obtained after LP (Figure 6e) and RCP (Figure 6f) radiation is incident. These micrographs of reflective color are consistent with the reflective spectra presented earlier (Figure $5)$.

Visibly transparent, near-IR reflective $\mathrm{CNC}$-organosilica photonic structures are prepared by increasing organosilica loading to red-shift both helicoidal optical components' reflection. The polarization-dependent nature of a single film compared to the polarization-independent reflection of its corresponding sandwich structure emulates our observations of the visibly reflective photonic crystal structures. Their reflection, $\mathrm{CD}$, and transmission spectra are obtained using the optical setup detailed in Figure 5a and d, respectively. A single reflective film has peak LP reflectivity $40.4 \%$ at $915.4 \mathrm{~nm}$ (Figure 7a) and comparatively shows a $97.0 \%$ increase in reflectivity when LCP radiation is incident, reaching $79.6 \%$ reflection at $915.4 \mathrm{~nm}$ (Figure 7a). When RCP radiation is incident upon a single reflective film, no reflection peak is observed and an average background reflectivity of $6.4 \%$ is captured (Figure 7a) due to reflection caused by the refractive index contrast at each of the film's optical interfaces with air. The IR-reflective sandwich structure's reflection is polarization independent, as a peak reflectivity of $83.6 \%$ at $895.6 \mathrm{~nm}$ is seen when LCP radiation is incident, comparable to peak reflectivity of $81.9 \%$ at the same wavelength when LP radiation is incident and peak reflectivity of $80.3 \%$ at $893.3 \mathrm{~nm}$ when RCP radiation is incident (Figure $7 b$ ). The CD signal of the sandwich structure confirms its polarization-independent interaction with light, as a weak CD signal is obtained, averaging $2.1 \%$ (Figure 7c). A single reflective film's CD spectrum shows a positive signal peaking at $72.5 \%$ at $915.4 \mathrm{~nm}$, revealing its highly polarization-dependent interaction with incident radiation (Figure 
7c). Visible transmission of the IR-reflective single film and sandwich structure yields comparable results, with a single film transmitting an average of $90.1 \%$ of incident visible radiation and the sandwich structure transmitting an average of $85.0 \%$ of visible radiation (Figure 7d). The 5.1\% difference between these two curves arises from the doubled number of optical interfaces of the sandwich structure compared to a single reflective film. Visibly transparent IR reflectors are of particular interest in applications such as solar gain regulators, radiative heat filters, microscopy optical elements, and other applications where optical observations need to take place alongside thermal shielding.

Numerical Modeling. Known analytical approximations ${ }^{23}$ do not take into account many of the experimental features present in our cholesteric assemblies and absorption of the material. To properly model propagation of light through our cholesteric-like structures, we use numerical modeling based on the Berreman $4 \times 4$ matrix method. ${ }^{26,27}$ This method allows one to calculate the reflectance of our cholesteric-like film in a broad spectral range while also taking into account cellulose's minimal absorption. The results of these numerical simulations are presented in Figure 8. Our numerical models are consistent with our experimental finding that the threelayer photonic structure allows for the near-100\% reflection of incident light due to their reflection of both handedness of circularly polarized radiation (Figure 8a).

Reflective efficiency depends on the thickness of the cholesteric-like films, which we cast in terms of the $\mathrm{d} / \mathrm{p}$ ratio, and also can be quantified by means of the integral reflectance $R_{\Sigma}=$ $\int_{\lambda_{1}}^{\lambda_{2}} R(\lambda) d \lambda$, where $R(\lambda)$ is the reflectance spectrum within a range $\lambda_{1}-\lambda_{2}$ at a given $d / p$. Numerical calculations show that reflective efficiency increases exponentially with the thickness of the film until it saturates at a film thickness equal to about 10 cholesteric pitches (Figure 8b), guiding our experimental efforts. The number of cholesteric pitches needed to saturate reflectance also depends on $\Delta n$ and increases as $\Delta n$ decreases. Calculations show that the reflective efficiency from the sandwich structure depends on the retardation of the CNC retardation plate and is maximized when $\Delta n d \approx \lambda / 2$ (Figure 8c). Our modeling reveals that the polarization-independent selective reflectivity of light can be designed to occur within a narrow part of the visible or nearIR spectral ranges (Figure 8a) or over a broader spectral range when cholesteric layers have nonzero pitch gradients across the film thickness. Importantly, this modeling also agrees with our experiments showing IR reflective films can be visibly transparent and, conversely, visibly 
reflective films can be IR-transparent, which is essential for many applications, including retrofittable window films for solar gain regulation.

\section{DISCUSSION}

Our findings illustrate that cholesteric-like photonic structures with high reflectivity can be obtained using the abundant, inexpensive biomaterial cellulose. The CNC-based tunable reflective sandwich films strongly interact with all polarizations of light, reflecting up to $83.6 \%$ of incident unpolarized radiation at wavelengths fulfilling the Bragg diffraction condition (with a full width at half-maximum of about $120 \mathrm{~nm}$ ), and are more than $85 \%$ transparent to radiation outside this range. Noteworthy, each CNC-based optical element composing the sandwich structure also serves as an individual optical element and may find separate applications. For example, nematic-like bacterial and cotton CNC assemblies operate as solid birefringent retarder plates with predesigned tunable phase retardation, which could be of interest for applications in polarizing optics and birefringent compensators used in liquid crystal displays. Preparing this structure entailed forming tunable, uniaxially aligned nematic-like CNC retardation plates via a series of repeated linear shear deposition cycles, kinetically preventing the CNC rods from selfassembling into their native cholesteric phase. Meanwhile, cholesteric-like dried CNC films function as tunable LCP light reflectors and as right-handed circular polarizers.

Self-assembly of nanocellulose into ordered optical structures increases their applied scope compared to conventional ordered nanostructures produced using top-down methods and, in doing so, promotes implementation in applications as diverse as smart clothes, document authenticity features, tunable inks, and visible-range transparent low-emissivity films suitable for retrofitting windows to boost their energy efficiency. From the standpoint of the latter application, various climates have different needs in terms of infrared radiation transmission and reflection to maximize building energy efficiency. In hot climates, it is desirable to reflect the sun's predominately near-IR radiation, thereby saving on air conditioning costs. Our near-IR reflecting films are suitable for this purpose. On the other hand, buildings in colder climates may save energy by letting the sun's near-IR radiation transmit through while benefiting from reflecting and trapping in the room's emission due to room-temperature blackbody radiation. Reflection of this predominantly mid-IR radiation (peaked at about $10 \mu \mathrm{m}$ ) could save energy 
and reduce the cost needed for building heating. Our current approach will need to be developed further to serve this need. During EISA, the colloidal CNCs dispersion's pitch shrinks about $90 \%$, from $\sim 6 \mu \mathrm{m}$ in the colloidal dispersion (with the exact pitch depending on CNC concentration, surface charge density, and processing conditions) to about $600 \mathrm{~nm}$ once fully dried. Therefore, a different approach, which could lock the cholesteric pitch at $6 \mu \mathrm{m}$ or so, is needed to fabricate mid-IR and other longer-wavelength reflective films. Such cholesteric-like mid-IR reflective films can be developed too, which will be reported elsewhere. In general, our cellulose-based multilayer films are suitable candidates for applications such as low-emissivity solar-gain-regulating window films that can be predesigned to give spectral reflectivity optimized for either hot or cold climates without compromising visible-range optical transparency.

\section{CONCLUSIONS AND OUTLOOK}

In this work we have numerically modeled, experimentally implemented, and optically characterized transparent and flexible laminar composite cholesteric- and nematic-like CNC based photonic structures with reflection tunable from visible to near-IR. We have shown that this sandwich structure features tunable, polarization-independent reflection approaching 100\% reflectivity, nearly double that compared to its corresponding single cholesteric-like CNC film within the visible and near-IR spectral ranges. The nematic-like CNC films' birefringence can be coarsely and finely tuned with bacterial and cotton CNC deposition. Such low-cost, highly reflective films made from renewable cellulose sources may find applications in thermally isolating viewports, economical color filters, 3D-printable pigments, document security features, and solar-gain-regulating technologies. Furthermore, its reflection may be further redshifted into the mid-IR regime by locking in its pitch prior to EISA, useful to trap heat in colder climates while still serving as visibly transparent window-retrofitting films with controlled solar gain.

\section{METHODS}

Bacterial cellulose pellicles served as one of our renewable cellulose sources to form CNCs and are obtained using Acetobacter hansenii cultured in Hestrin-Schramm medium at $26^{\circ} \mathrm{C}$ for 3 
weeks with a dry mass yield of $7.5 \mathrm{~g} / \mathrm{L}$. In situ darkfield microscopy reveals cellulose is formed at solid/liquid and gas/liquid interfaces and that the bacteria excrete cellulose along their length. During this process neighboring secreted nanofibers hydrogen bond to one another, forming ribbon-shaped cellulosic assemblies hundreds of micrometers in length, with a cross-sectional area of $\sim 10 \mathrm{~nm}$ by $\sim 50 \mathrm{~nm} .{ }^{28}$ Cellulose production also serves as a bacteria propulsion mechanism, and cellulose-ejecting bacteria move as fast as $6 \mu \mathrm{m} / \mathrm{min}$ in the direction opposite the newly ejected cellulosic ribbon. Other bacteria can glide on established cellulose ribbons (Figure 9a) at about the same speed. After cellulose secretion, bacteria detach from the cellulose fibrils (Figure 9b). At some critical cellulosic ribbon concentration, the ribbons begin to physically cross-link via hydrogen bonds, forming an extended network (Figure 9c) and eventually an entire cellulose pellicle.

Colloidal CNCs are prepared from bacterial cellulose and cotton balls (Swisspers brand). Raw bacterial cellulose pellicles are purified by soaking in $1 \mathrm{wt} \%$ sodium hydroxide solution at $70{ }^{\circ} \mathrm{C}$ for $1 \mathrm{~h}$, followed by extensive washing with distilled water and drying at ambient conditions. The cotton balls are used without further purification. The cellulose source is hydrolyzed to yield CNCs by modifying the protocol in the literature. ${ }^{13}$ Briefly, the cellulose is mechanically stirred at $1000 \mathrm{rpm}$ in $64 \mathrm{wt} \%$ sulfuric acid at $45^{\circ} \mathrm{C}$ with an optimized celluloseto-acid mass ratio of 1:25 for a preset time ( $1 \mathrm{~h}$ for bacterial cellulose and $2 \mathrm{~h}$ for cotton cellulose, respectively) assisted by continuous sonication in a bath sonicator (Branson 3800). After the reaction, $\mathrm{CNCs}$ are decorated with negatively charged sulfate ester moieties substituting about $5 \%$ of primary alcohols present along the CNCs' main chain backbone (Figure 9d) ${ }^{29}$ Colloidal CNCs are then purified by aqueous dilution and repeated washing via centrifugation at 10k g-force for 8 min intervals. The supernatant is then dialyzed against deionized water using a membrane with a cutoff molecular weight of 12 000-14 000 g/mol until the $\mathrm{pH}$ of the dialysis water reached 7 , followed by brief sonication, centrifuged at $10000 \mathrm{~g}$ force for 8 min one last time to remove remaining microsized $\mathrm{CNC}$ aggregations, and then finally filtrated through $11 \mu \mathrm{m}$ pores (Whatman no. 1 filter paper) for bacterial CNCs and $5 \mu \mathrm{m}$ pores (Millipore MF filters) for cotton CNCs. The study detailed herein utilizes both bacterialand cotton-derived nanocellulose, with CNCs measuring approximately $50 \mathrm{~nm}$ by $2 \mu \mathrm{m}$ (Figure 9e) and $10 \mathrm{~nm}$ by $200 \mathrm{~nm}$ (Figure 9f), respectively.

Composite cotton $\mathrm{CNC}-$ organosilica solid reflective films are prepared by modifying the 
protocol previously reported in the literature,30 using organosilica precursor 1,2-bis(trimethoxysilyl)ethane (96\% pure, Alfa Aesar), and with addition of polyethylene glycol (number-average molecular weight 400) prior to $1 \mathrm{~h}$ of magnetic stirring at $600 \mathrm{rpm}$ of the organosilica precursor with aqueous CNCs. The film is cast on a polystyrene Petri dish and cured in ambient conditions in the presence of a $2000 \mathrm{G}$ magnetic field oriented normal to the film surface, to align the helical axis uniformly perpendicular to the film surface. After evaporation, the solid freestanding film preserves the cholesteric-like configuration with an accompanying pitch shrinkage. The four reflective cholesteric-like $\mathrm{CNC}-$ organosilica films detailed herein have the composition listed in Table 1.

The solid nematic-like bacterial or cotton CNC retardation plate is prepared by manual linear shear deposition of aqueous 3.5-5.0 wt \% bacterial CNCs or 3.5-5.0 wt \% cotton CNCs on a glass plate or directly on top of a cholesteric-like $\mathrm{CNC}$ film at a rate of $0.25 \mathrm{~cm} / \mathrm{s}$, followed by drying on a $30-35{ }^{\circ} \mathrm{C}$ hot plate. ${ }^{31}$ This process is repeated until the desired half-wavelength retardation is achieved. When shear depositing on a glass plate, the nematic-like retarder film was removed from the glass plate by shearing with a cutting edge, leaving a freestanding film. All sandwich structures detailed herein were prepared by linear shear deposition of aqueous CNC directly on one cholesteric-like CNC-based film. To form the final sandwich structure from this two-layered film, another single cholesteric-like CNC-based reflective film is adhered to the two-layered film, with the nematic-like layer in the center, using a thin layer of adhesive (NOA 65, Norland Products, Inc.), followed by 1 min of $365 \mathrm{~nm}$ wavelength curing (OmniCure Series 2000).

CNCs' morphology and geometry are elucidated by transmission electron microscopy (FEI Philips CM100) operating at $80 \mathrm{kV}$. The uniaxially aligned nematic-like CNC layer's structure is determined via scanning electron microscopy (Carl Zeiss EVO MA 10) operating at $5 \mathrm{kV}$. Optical transmission, reflection, and CD spectra are obtained at normal incidence with an Ocean Optics USB2000+ spectrometer in conjunction with an Olympus BX-51 microscope equipped with a linear polarizer, an appropriate achromatic quarter-wave plate (Thorlabs, Inc., AQWP05M-600 or AQWP05M-980), and an Olympus LMPLFLN 20× objective. Polarized optical micrographs are captured via CCD (Point Grey Research, GS3- U3-28S5C). CNC orientation in the optical retardation layer is deduced with the insertion of a $530 \mathrm{~nm}$ retardation plate, and its retardation is quantified with a Berek compensator (Olympus U-CTB). 


\section{ACKNOWLEDGMENTS}

This research was supported by the U.S. Department of Energy, Advanced Research Projects Agency-Energy award DEAR0000743. We thank Eugenia Kumacheva for insightful discussions and David A. Rudman and Yao Zhai for technical assistance.

\section{REFERENCES}

(1) Datta, T.; Sen, M. Characterization of slotted photonic crystal waveguide and its application in nonlinear optics. Superlattices Microstruct. 2017, 109, 107-116.

(2) Travers, J. C.; Rulkov, A. B.; Cumberland, B. A.; Popov, S. V.; Taylor, J. R. Visible supercontinuum generation in photonic crystal fibers with a $400 \mathrm{~W}$ continuous wave fiber laser. Opt. Express 2008, 16, 14435-14447.

(3) Yoon, B.; Lee, J.; Park, I. S.; Jeon, S.; Lee, J.; Kim, J.-M. Recent functional material based approaches to prevent and detect counterfeiting. J. Mater. Chem. C 2013, 1, 2388-2403.

(4) Hu, H.; Chen, Q.-W.; Tang, J.; Hu, X.-Y.; Zhou, X.-H. Photonic anti-counterfeiting using structural colors derived from magneticresponsive photonic crystals with double photonic bandgap heterostructures. J. Mater. Chem. 2012, 22, 11048-11053.

(5) Kuang, M.; Wang, J.; Bao, B.; Li, F.; Wang, L.; Jiang, L.; Song, Y. Inkjet printing patterned photonic crystal domes for wide viewingangle displays by controlling the sliding three phase contact line. Adv. Opt. Mater. 2014, 2, 34-38.

(6) Lee, S. Y.; Kim, S.-H.; Hwang, H.; Sim, J. Y.; Yang, S.-M. Controlled pixelation of inverse opaline structures towards reflectionmode displays. Adv. Mater. 2014, 26, 2391-2397.

(7) Vukusic, P.; Sambles, J. R. Photonic structures in biology. Nature 2004, 424, 852-855.

(8) Finlayson, E. D.; McDonald, L. T.; Vukusic, P. Optically ambidextrous circularly polarized reflection from the chiral cuticle of the scarab beetle Chrysina resplendens. J. R. Soc., Interface 2017, 14, 20170129.

(9) Fernandes, S. N.; Almeida, P. L.; Monge, N.; Aguirre, L. E.; Reis, D.; de Oliveira, C. L. P.; Neto, A. M. F.; Pieranski, P.; Godinho, M. H. Mind the microgap in iridescent cellulose nanocrystal films. Adv. Mater. 2017, 29, 1603560. 
(10) Lawrence, J. R.; Ying, Y.; Jiang, P.; Foulger, S. H. Dynamic tuning of organic lasers with colloidal crystals. Adv. Mater. 2006, 18, 300-303.

(11) Liu, Y.; Qin, F.; Wei, Z.-Y.; Meng, Q.-B.; Zhang, D.-Z.; Li, Z.-Y. 10 fs ultrafast all-optical switching in polystyrene nonlinear photonic crystals. Appl. Phys. Lett. 2009, 95, 131116.

(12) Habibi, Y.; Lucia, L. A.; Rojas, O. J. Cellulose nanocrystals: chemistry, self-assembly, and applications. Chem. Rev. 2010, 110, 3479-3500.

(13) Giese, M.; Blusch, L. K.; Khan, M. K.; MacLachlan, M. J. Functional materials from cellulose-derived liquid-crystal templates. Angew. Chem., Int. Ed. 2015, 54, 2888-2910.

(14) Hiratani, T.; Hamad, W. Y.; MacLachlan, M. J. Transparent depolarizing organic and inorganic films for optics and sensors. Adv. Mater. 2017, 29, 1606083.

(15) Hwang, J.; Song, M. H.; Park, B.; Nishimura, S.; Toyooka, T.; Wu, J. W.; Takanishi, Y.; Ishikawa, K.; Takezoe, H. Electro-tunable optical diode based on photonic bandgap liquidcrystal heterojunctions. Nat. Mater. 2005, 4, 383-387.

(16) Yang, D.-K.; Huang, X.-Y.; Zhu, Y.-M. Bistable cholesteric reflective displays: materials and drive schemes. Annu. Rev. Mater. Sci. 1997, 27, 117-146.

(17) Comoretto, D. Organic and Hybrid Photonic Crystals; Springer, 2015.

(18) Marchessault, R. H.; Morehead, F. F.; Walter, N. M. Liquid crystal systems from fibrillar polysaccharides. Nature 1959, 184, 632- 633.

(19) Onsager, L. The effects of shape on the interaction of colloidal particles. Ann. N. Y. Acad. Sci. 1949, 51, 627-659.

(20) Gray, D. G. Isolation and handedness of helical coiled cellulosic thickenings from plant petiole tracheary elements. Cellulose 2014, 21, 3181-3191.

(21) Majoinen, J.; Kontturi, E.; Gray, D. G. SEM imaging of chiral nematic films cast from cellulose nanocrystal suspensions. Cellulose 2012, 19, 1599-1605.

(22) Mitov, M. Cholesteric liquid crystals with a broad light reflection band. Adv. Mater. 2012, 24, 6260-6276.

(23) Yang, D.-K.; Wu, S.-T. Fundamentals of Liquid Crystal Devices; Wiley, 2014.

(24) Brinker, C. J.; Lu, Y.; Sellinger, A.; Fan, H. Evaporation-induced self-assembly: nanostructures made easy. Adv. Mater. 1999, 11, 579- 585.

(25) Pan, J.; Hamad, W. Y.; Straus, S. K. Parameters affecting the chiral nematic phase of nanocrystalline cellulose films. Macromolecules 2010, 43, 3851-3858. 
(26) Berreman, D. W. Optics in stratified and anisotropic media: $4 \times 4$-matrix formulation. J. Opt. Soc. Am. 1972, 62, 502-510.

(27) Stallinga, S. Berreman $4 \times 4$ matrix method for reflective liquid crystal displays. J. Appl. Phys. 1999, 85, 3023-3031.

(28) Moon, R. J.; Martini, A.; Nairn, J.; Simonsen, J.; Youngblood, J. Cellulose nanomaterials review: structure, properties and nanocomposites. Chem. Soc. Rev. 2011, 40, 3941-3994.

(29) Hamad, W. Y.; Hu, T. Q. Structure-process-yield interrelations in nanocrystalline cellulose extraction. Can. J. Chem. Eng. 2010, 88, 392-402.

(30) Shopsowitz, K. E.; Hamad, W. Y.; MacLachlan, M. J. Flexible and iridescent chiral nematic mesoporous organosilica films. J. Am. Chem. Soc. 2012, 134, 867-870.

(31) Liu, Q.; Campbell, M. G.; Evans, J. S.; Smalyukh, I. I. Orientationally ordered colloidal codispersions of gold nanorods and cellulose nanocrystals. Adv. Mater. 2014, 26, 7178-7184. 

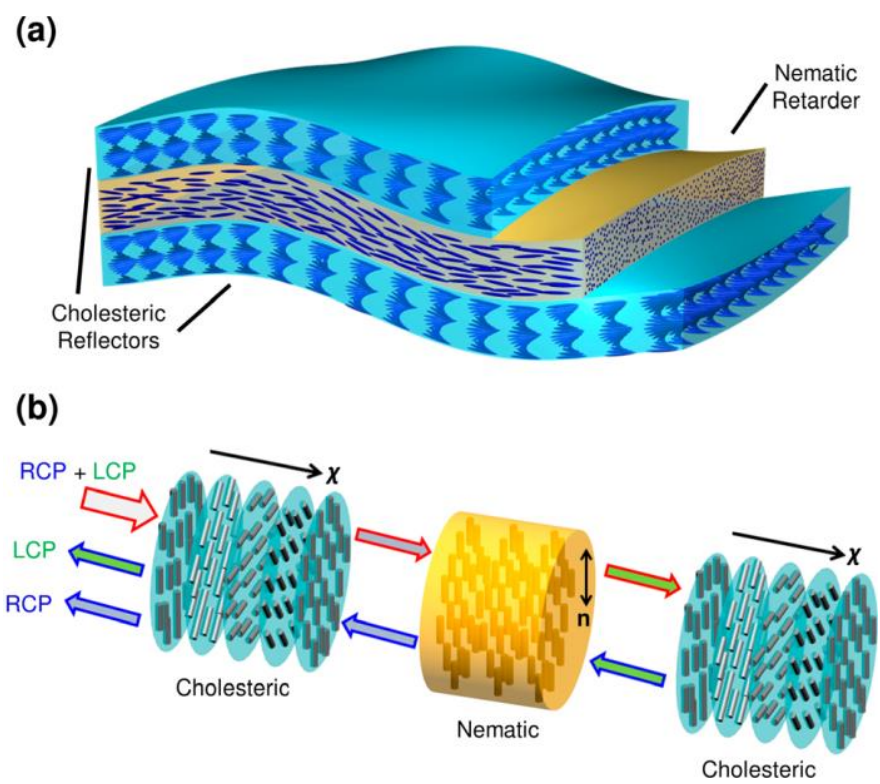

Figure 1. Schematics detailing (a) the proposed sandwich structure's architecture and flexibility and (b) its cross-section depicting the mechanism enabling its polarization-independent selective Bragg reflection. The small rods represent individual CNCs (not to scale). 

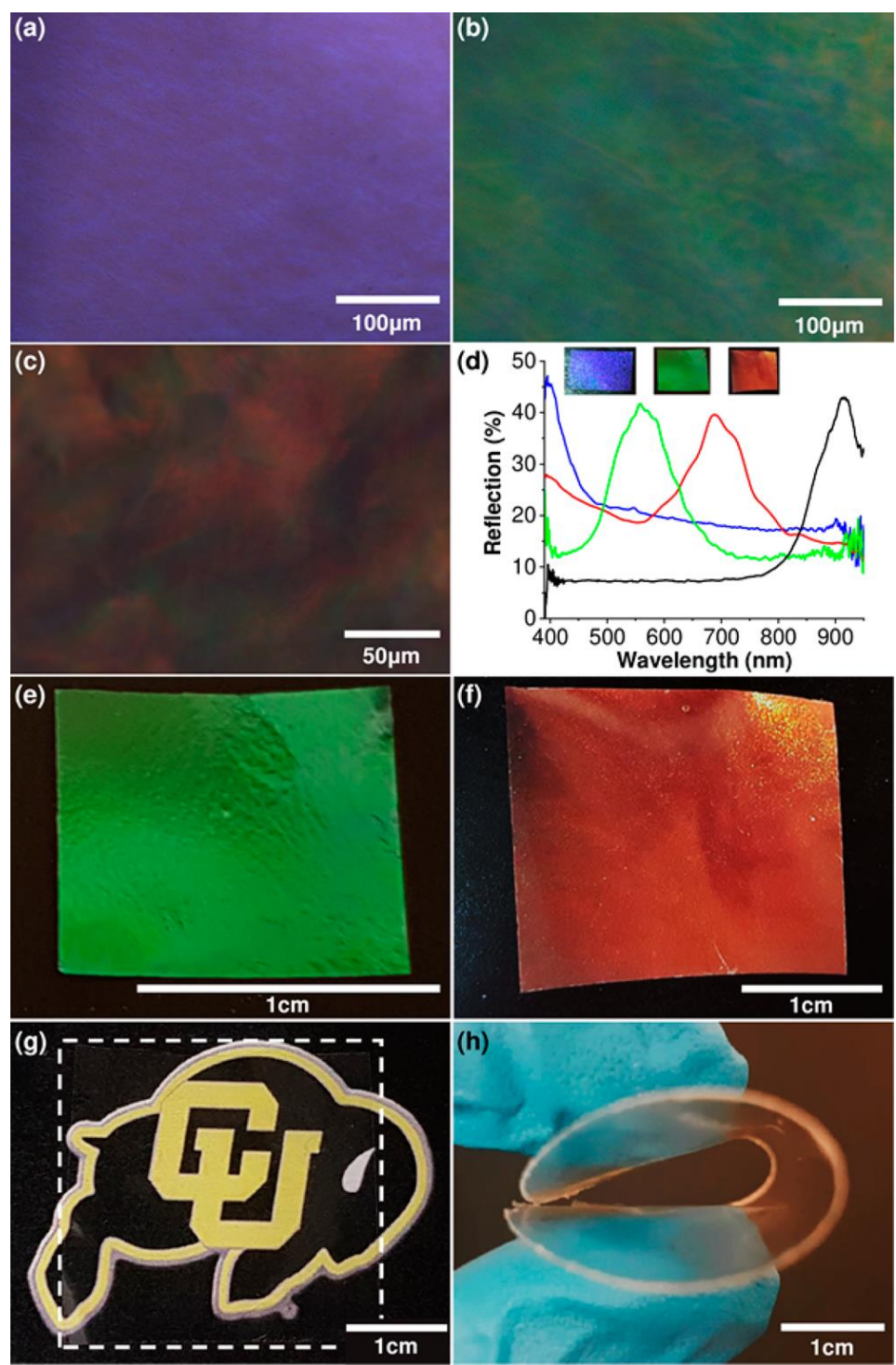

Figure 2. Reflective optical micrographs with natural light incident upon CNC-based reflective films with reflection peaks centered at (a) $400 \mathrm{~nm}$ (b) $557 \mathrm{~nm}$, and (c) $688 \mathrm{~nm}$ prepared with organosilica loading ranging from 21.3 to $32.6 \mathrm{wt} \%$ in the presence of a static $2000 \mathrm{G}$ magnetic field directed parallel to the surface normal. In (d) these films' reflection spectrum is plotted in their predominant reflective color. The near-IR reflective film's reflectivity peaks at $915 \mathrm{~nm}$ and is plotted in black. In the inset, a $2 \mathrm{~cm}$ wide blue, $1.15 \mathrm{~cm}$ wide green, and $2 \mathrm{~cm}$ wide red reflective cholesteric-like film are shown. (e) Image of the green-reflective cholesteric-like film. (f) Image of the red-reflective cholesteric-like film. In (g) a highly visibly transparent near- IR reflective film is shown, with its perimeter outlined using white dashed marks. (h) Demonstration of flexibility of the film enabled by the addition of $30 \mathrm{wt} \%$ of the plasticizer PEG-400. 


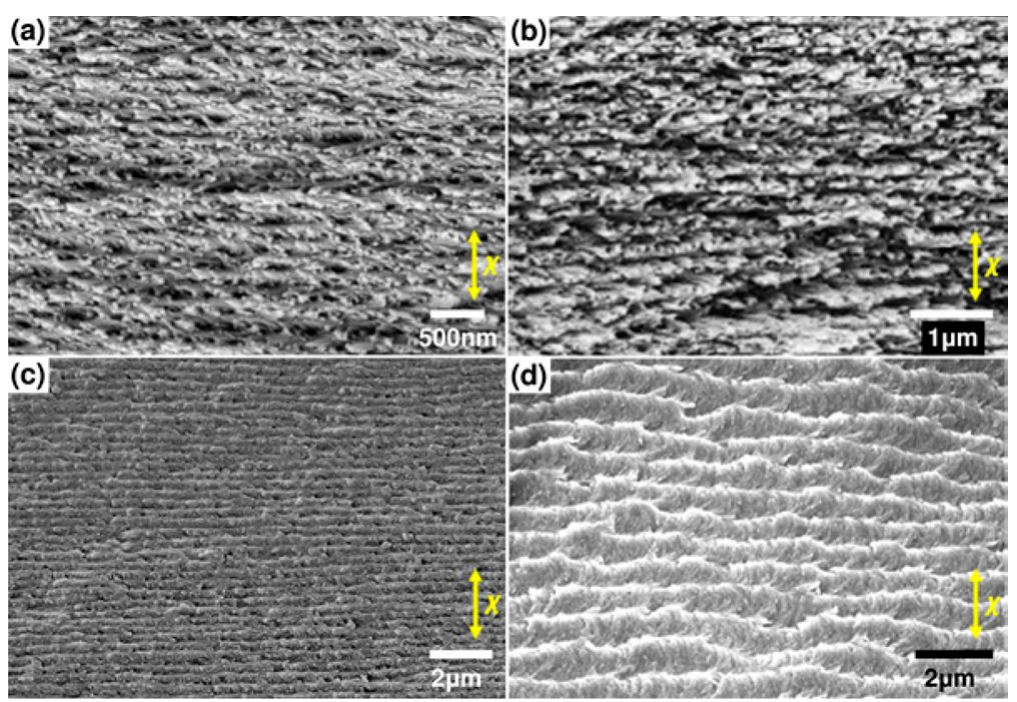

Figure 3. Cross-sectional scanning electron micrographs of dried CNC-based cholesteric-like films confirm effectiveness of static $2000 \mathrm{G}$ magnetic field alignment in promoting macroscopic single-domain configurations. The pitch, and thus the corresponding reflection peak, is tunable from $p=$ (a) $286 \mathrm{~nm}$ to (b) $357 \mathrm{~nm}$ to (c) $660 \mathrm{~nm}$ to (d) $1.3 \mu \mathrm{m}$. 

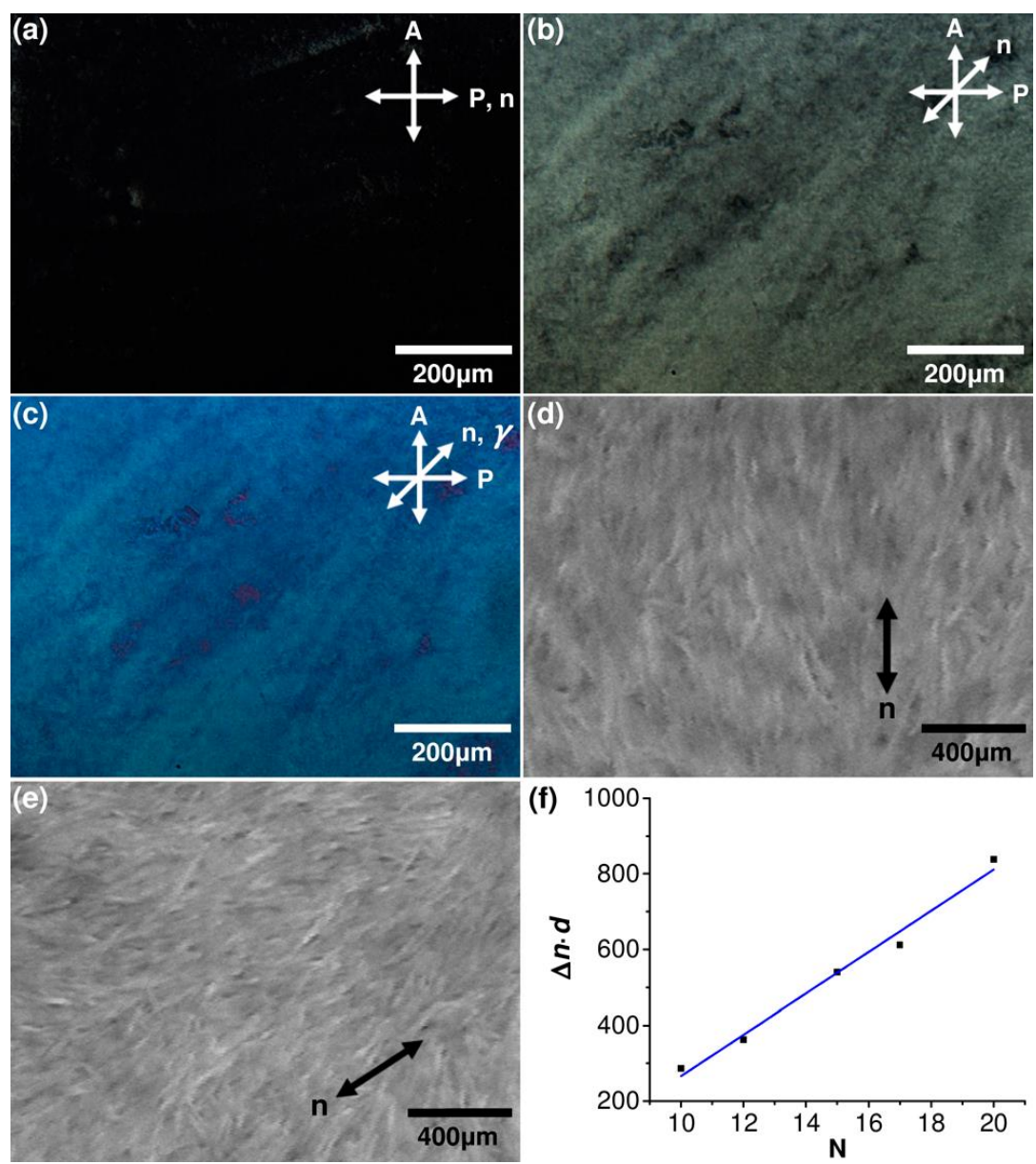

Figure 4. Polarized optical micrographs of the CNC-based retardation plates with the director oriented (a) parallel to the polarizer and (b, c) $+45^{\circ}$ with respect to the polarizer (b) before and (c) after insertion of a $530 \mathrm{~nm}$ retardation plate. Scanning electron micrographs taken along the direction normal to the shear plane for the films with both (d) bacterial CNCs and (e) cotton $\mathrm{CNCs}$, showing unidirectional alignment along the shear direction. The $\mathrm{CNC}$ director has orientation denoted by the double-headed arrow labeled n. (f) Linear relation of $\Delta n d$ vs the number of deposited CNC layers $N$ of a unidirectionally aligned nematic-like bacterial CNC wave plate. 
(a)

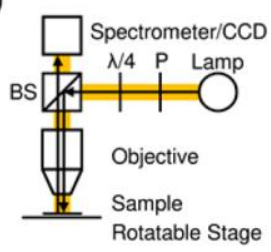

(d)

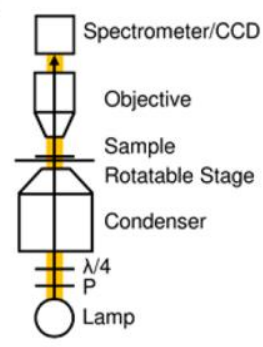

(b)

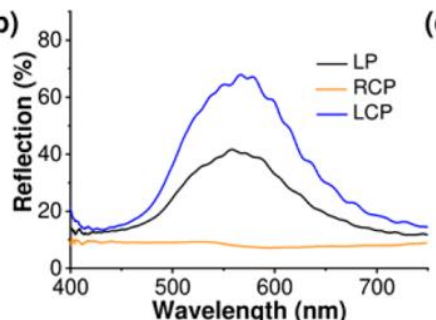

(e) 0.8 . - Single Film

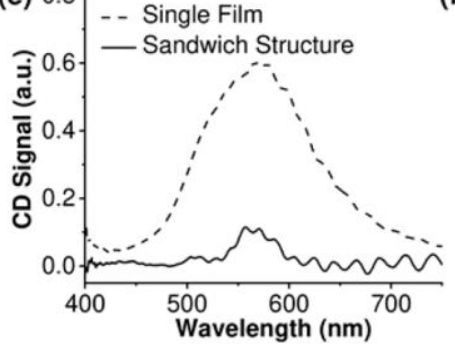

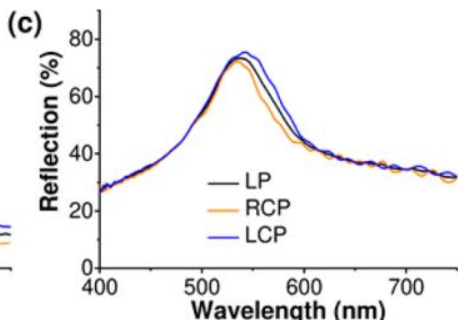

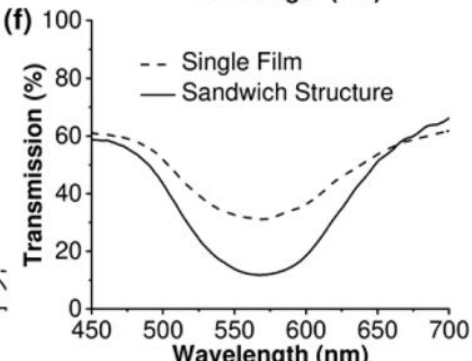

Figure 5. (a) Optical setup used to characterize reflection and CD spectra of both single-layer reflective films and their corresponding sandwich structure. $\mathrm{P}$ is a linear polarizer, $\lambda / 4$ is an achromatic quarter-wave plate, and BS is a beam splitter. (b) Optical characterization of a visible-range single reflective film and its corresponding sandwich structure. The reflection spectrum of a single reflective cotton-based CNC-organosilica film with LP, RCP, and LCP incident light. (c) Corresponding reflection spectra of a visibly reflective sandwich structure. (d) Optical setup used to characterize transmission spectra of both single reflective films and their corresponding sandwich structure. (e) CD spectra of both the single film and the sandwich structure. (f) Transmission spectra with natural light incident on a single film and its analogous sandwich structure.

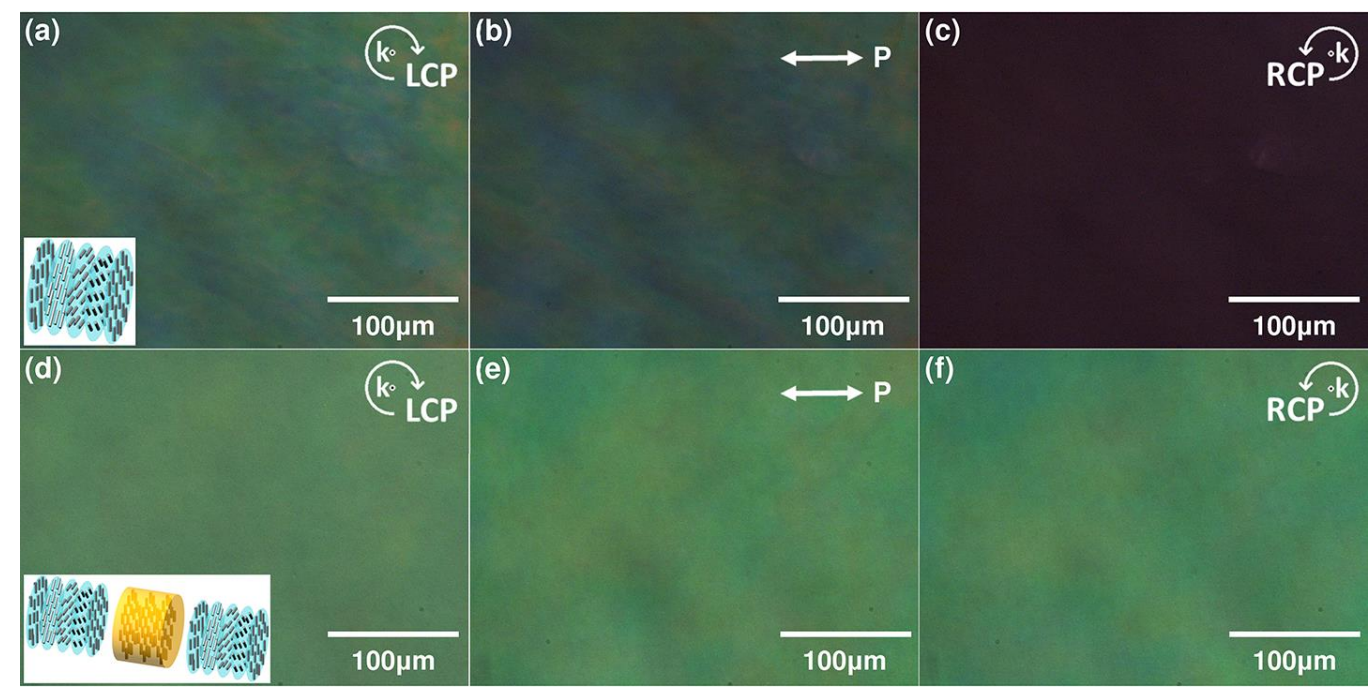

Figure 6. Reflective optical micrographs of a single composite cotton CNC-organosilica 
reflective film (top row) with incident (a) LCP, (b) LP, and (c) RCP radiation and of its corresponding sandwich structure (bottom row), as seen with the incident (d) LCP, (e) LP, and (f) RCP light. LCP and RCP are respectively denoted by clockwise and counterclockwise circular arrows centered about a wave vector $\mathrm{k}$ parallel to $\chi$.
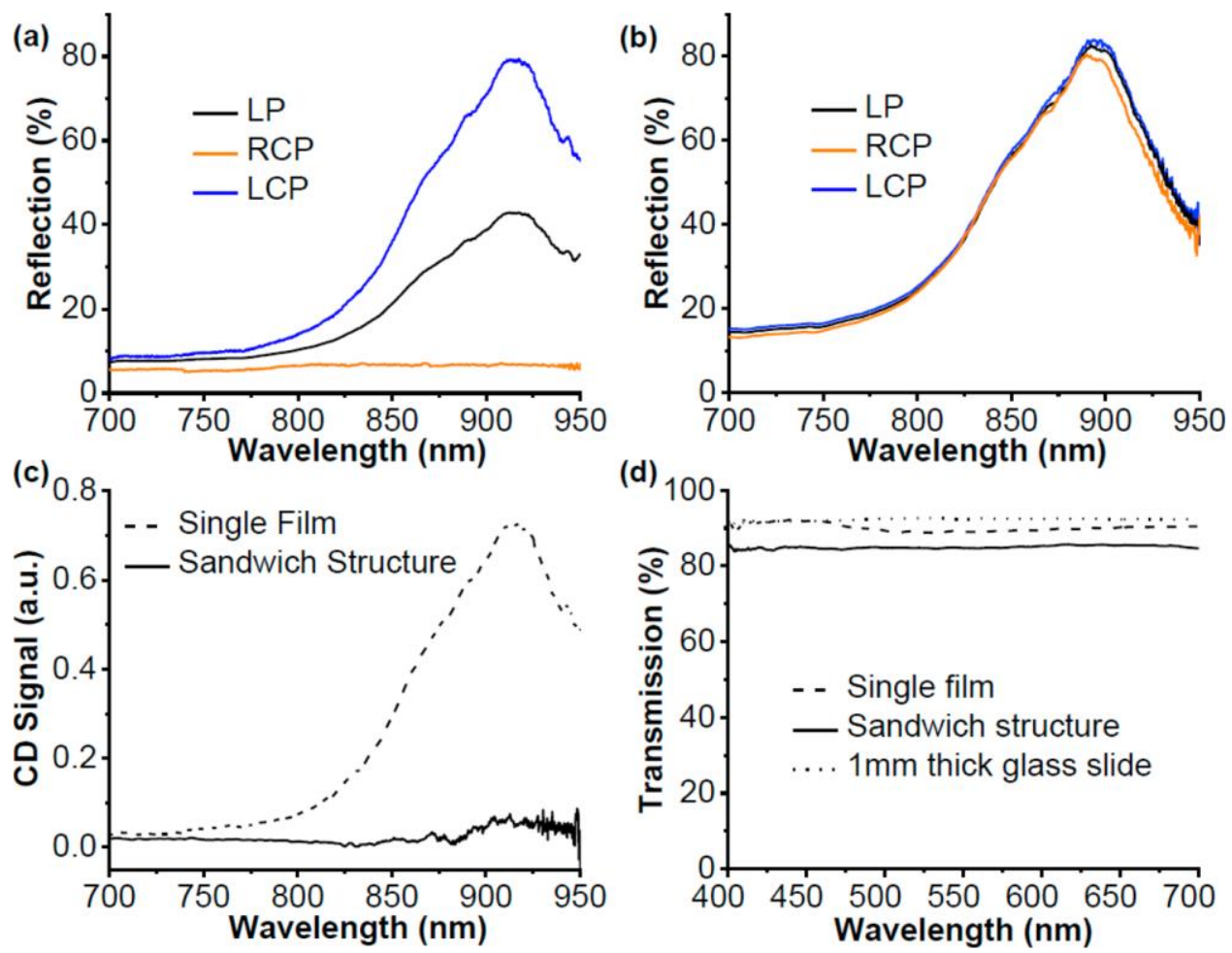

Figure 7. Optical characterization of a visibly transparent, single near-IR reflective film and its corresponding sandwich structure. (a) Reflection spectrum of a single reflective cotton CNCorganosilica film with incident LP, RCP, and LCP radiation. (b) Corresponding reflection spectra of a sandwich structure. (c) CD spectra of both the single film and the sandwich structure. (d) Transmission spectra with natural light incident upon a single film and its corresponding sandwich structure, showing average visible transmission of $90.1 \%$ for a single reflective film and $85.0 \%$ for the corresponding sandwich structure. The transmission of a $1 \mathrm{~mm}$ thick glass slide, averaging $92.3 \%$ visible transmission, is provided for comparison. 

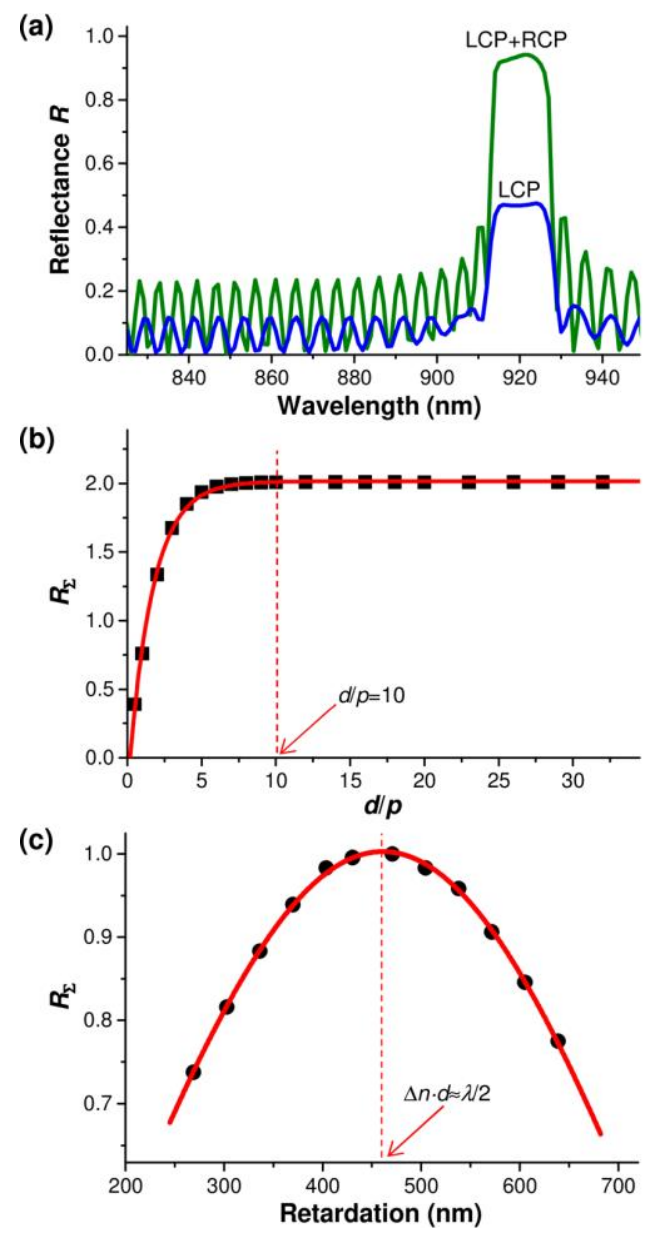

Figure 8. (a) Computer-simulated reflection for near-IR reflective photonic structures, showing a theoretically doubled reflection of a sandwich structure (plotted in green) as compared to its single film counterpart (plotted in blue). (b) Reflective efficiency's dependency on the film thickness-to-pitch (d/p) ratio. (c) Reflective efficiency of the three-layer composite structure shown in (a) depends on the effective retardation of the CNC retardation plate and is maximized when $\Delta n d \approx \lambda / 2$. 

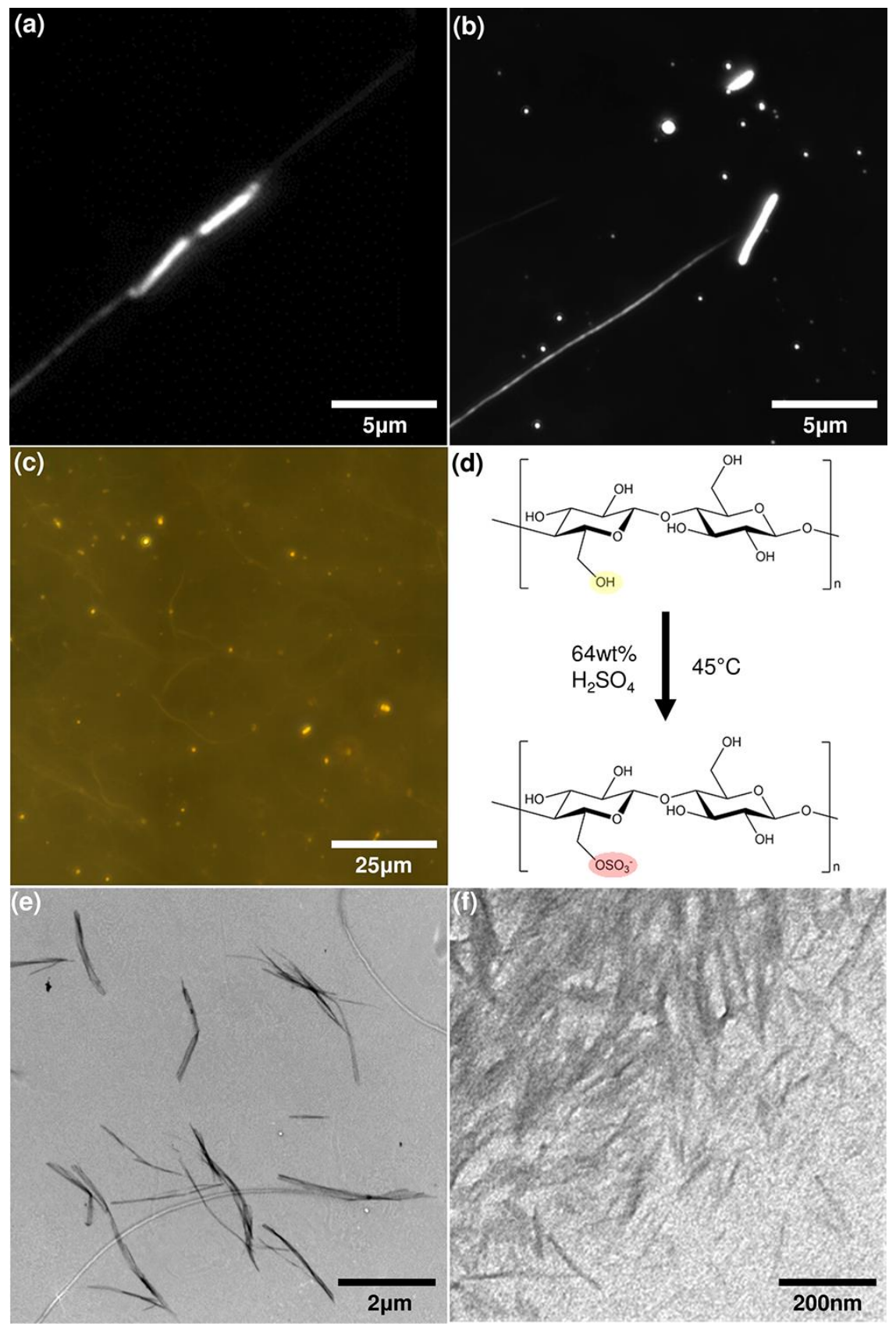

Figure 9. Dark-field micrographs of bacterium Acetobacter hansenii (a) traversing along a cellulose fibril and (b) detaching from an excreted cellulose ribbon, freely moving within the culture medium. (c) Several Acetobacter hansenii bacteria embedded within the loosely physically cross-linked cellulosic network. (d) During CNC synthesis, some portion of the cellulose's primary alcohols (located on C6, highlighted in yellow) are sulfated, leaving the rod with a net negative charge (highlighted in red). Transmission electron micrographs of (e) bacterial CNCs and (f) cotton CNCs. 
Table 1. Composition of CNC-Organosilica Reflective Films with Predesigned Reflective Colors

\begin{tabular}{|l|c|c|c|c|}
\hline \multirow{2}{*}{ Component } & \multicolumn{4}{|c|}{ Reflective color } \\
\cline { 2 - 5 } & Blue & Green & Red & Near-IR \\
\hline cotton CNC (wt \%) & 48.7 & 46.0 & 43.9 & 37.4 \\
\hline organosilica (wt \%) & 21.3 & 24.0 & 26.1 & 32.6 \\
\hline PEG-400 (wt \%) & 30.0 & 30.0 & 30.0 & 30.0 \\
\hline
\end{tabular}

